\title{
High-efficiency, ultrafast separation of emulsified oil-water mixtures
}

\author{
Arun K Kota and Anish Tuteja \\ NPG Asia Materials (2013) 5, e58; doi:10.1038/am.2013.34; published online 16 August 2013
}

Multiple oil spill disasters over the last few years have highlighted the challenges of effective oil-water separation. The separation of oil-water micro- and nano-emulsions (emulsions with dispersed droplet sizes in the micro- or nano-meter range) can be particularly difficult. ${ }^{1,2}$ Shi et al. ${ }^{3}$ from the Chinese Academy of Sciences in Suzhou and Beijing have now developed ultrathin carbon nanotube membranes that can separate a wide range of oil-water micro- and nano-emulsions with separation efficiency $>99.9 \%$. Perhaps more significantly, the separation fluxes are 2-3 orders of magnitude higher than those obtained with current commercially available separation membranes.

Membrane-based oil-water separation technologies are attractive for emulsion separation because they are energy-efficient, cost-effective and applicable across a wide range of industrial effluents. One factor that governs the flux of the permeate during separation is the membrane thickness. This separation flux is inversely proportional to the thickness of the membrane, that is, thinner membranes provide a higher separation flux. The thickness of commercially available separation membranes can vary between tens and hundreds of micrometers. In comparison, Shi et al. ${ }^{3}$ developed free-standing single-walled carbon nanotube network films that can be as thin as 30 nanometers. These films are hydrophobic (water-repellent with water contact angles $>90^{\circ}$ ) and superoleophilic (extremely oil-absorbent with oil contact angles of $0^{\circ}$ ). Consequently, they allow oil to easily permeate through, while simultaneously preventing the permeation of water. Multiple groups have previously developed hydrophobic and oleophilic surfaces, ${ }^{4,5}$ however the membranes developed by Shi et $a l^{3}$ are perhaps the thinnest to date. By utilizing these ultrathin membranes in a separation apparatus (Figure 1a), Shi et al. ${ }^{3}$ demonstrated the separation of various oil-water emulsions with fluxes between $10^{4}-10^{5} \mathrm{~L} \mathrm{~m}^{-2} \mathrm{~h}^{-1}$ per bar, which are $2-3$ orders of magnitude higher

Department of Materials Science and Engineering, University of Michigan, Ann Arbor, MI, USA

E-mail: atuteja@umich.edu
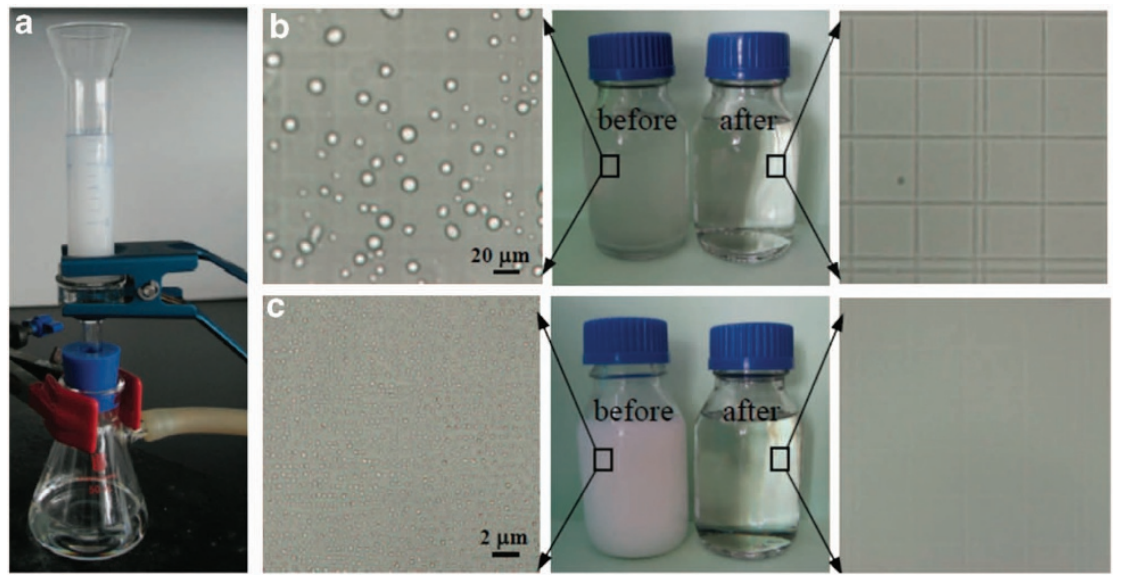

Figure 1 (a) A separation apparatus with the filtration cell sandwiched between glass beakers. ( $b$ and c) A water-in-petroleum ether microemulsion and a water-in-toluene nanoemulsion, respectively, before separation, and the corresponding permeates after separation. Copyright Wiley-VCH, 2013.

than the fluxes typically obtained with commercial separation membranes.

Their separation apparatus consisted of a filtration cell (ultrathin carbon nanotube membrane placed on a ceramic membrane) sandwiched between two glass beakers (Figure 1a). When a water-in-oil emulsion was added above the filtration cell, and a transmembrane pressure applied, oil permeated through the membrane, while water was retained above the membrane. In this manner, a series of water-in-oil emulsions, including surfactant-free water-in-petroleum ether, water-in-isooctane, water-in-cyclohexane microemulsions (Figure 1b) and span80-stabilized water-in-toluene nanoemulsions (Figure 1c), were separated with a separation efficiency $>99.9 \%$.

The chemical stability of carbon nanotubes against a range of acids and bases allows these membranes to separate a broad range of water-inoil emulsions. One potential limitation of the membranes is that they can get fouled by the oil present within the emulsions, and therefore have to be periodically cleaned by passing ethanol. Further research on effectively separating commercially relevant emulsions and oil-in-water emulsions using these membranes, as well as, improving their anti-fouling properties can make these membranes even more attractive for industrial deployment.

1 Cheryan, M. \& Rajagopalan, N. Membrane processing of oily streams. Wastewater treatment and waste reduction. J. Memb. Sci. 151, 13-28 (1998).

2 Kota, A. K., Kwon, G., Choi, W., Mabry, J. M. \& Tuteja, A. Hygro-responsive membranes for effective oil-water separation. Nat. Commun. 3, 1025 (2012).

3 Shi, Z., Zhang, W., Zhang, F., Liu, X., Wang, D., Jin, J. \& Jiang, L. Ultrafast separation of emulsified oil/water mixtures by ultrathin free-standing single-walled carbon nanotube network films. Adv. Mater. 25, 2422-2427 (2013).

4 Feng, L., Zhang, Z., Mai, Z., Ma, Y., Liu, B., Jiang, L. \& Zhu, D. A super-hydrophobic and super-oleophilic coating mesh film for the separation of oil and water. Angew. Chem. Int. Ed. 43, 2012-2014 (2004).

5 Yuan, J., Liu, X., Akbulut, O., Hu, J., Suib, S. L., Kong, J. \& Stellacci, F. Superwetting nanowire membranes for selective absorption. Nat. Nanotechnol. 3, 332-336 (2008).

(c) (i) $\odot$ This work is licensed under a Creative Commons AttributionNonCommercial-NoDerivs 3.0 Unported License. To view a copy of this license, visit http:// creativecommons.org/licenses/by-nc-nd/3.0/ 